\title{
Texture Analysis in the Assessment of Rectal Cancer: Comparison of T2WI and Diffusion-Weighted Imaging
}

\author{
Ming Li, ${ }^{1}$ Xiaodan Xu, ${ }^{2}$ Pengjiang Qian, ${ }^{3}$ Heng Jiang, ${ }^{4}$ Jianlong Jiang, ${ }^{1}$ Jinbing Sun $\mathbb{D}^{1}{ }^{1}$ \\ and Zhihua Lu $\mathbb{D D}^{4}$ \\ ${ }^{1}$ Department of General Surgery, Changshu No. 1 People's Hospital, Changshu, 215500 Jiangsu Province, China \\ ${ }^{2}$ Department of Gastroenterology, Changshu No. 1 People's Hospital, Changshu, 215500 Jiangsu Province, China \\ ${ }^{3}$ School of Artificial Intelligence and Computer Science, Jiangnan University, Wuxi, 214122 Jiangsu Province, China \\ ${ }^{4}$ Department of Radiology, Changshu No. 1 People's Hospital, Changshu, 215500 Jiangsu Province, China
}

Correspondence should be addressed to Jinbing Sun; sunjb515@163.com and Zhihua Lu; luzhihua4100@suda.edu.cn

Received 22 March 2021; Revised 5 July 2021; Accepted 27 August 2021; Published 15 September 2021

Academic Editor: Alireza Jolfaei

Copyright ( 92021 Ming Li et al. This is an open access article distributed under the Creative Commons Attribution License, which permits unrestricted use, distribution, and reproduction in any medium, provided the original work is properly cited.

Texture analysis (TA) techniques derived from T2-weighted imaging (T2WI) and apparent diffusion coefficient (ADC) maps of rectal cancer can both achieve good diagnosis performance. This study was to compare TA from T2WI and ADC maps between different pathological $\mathrm{T}$ and $\mathrm{N}$ stages to confirm which TA analysis is better in diagnosis performance. 146 patients were enrolled in this study. Tumor TA was performed on every patient's T2WI and ADC maps, respectively; then, skewness, kurtosis, uniformity, entropy, energy, inertia, and correlation were calculated. Our results demonstrated that those significant different parameters derived from T2WI had better diagnostic performance than those from ADC maps in differentiating pT3b-4 and pN1-2 stage tumors. In particular, the energy derived from T2WI was an optimal parameter for diagnostic efficiency. Highresolution T2WI plays a key point in the local stage of rectal cancer; thus, TA derived from T2WI may be a more useful tool to aid radiologists and surgeons in selecting treatment.

\section{Introduction}

The incidence and mortality of colorectal cancer in China have maintained an upward trend, which ranks third and fifth among all malignant tumors. Among them, rectal cancer accounts for half of colorectal cancer in China. [1].

Pathological $\mathrm{T}$ and $\mathrm{N}$ stages of rectal cancer were important prognostic factors affecting patients [2]. The patients of rectal cancer with lymph node metastasis require neoadjuvant chemotherapy. pT1-2 stage patients do not require neoadjuvant chemotherapy due to a low recurrence rate; conversely, pT3-4 stage patients need neoadjuvant chemotherapy $[3,4]$. The prognosis of pT3 stage rectal cancer varies greatly based on extramural depth (EMD) of tumor invasion [5]. The $5 \mathrm{~mm}$ cutoff of EMD has been determined to be the most discriminating and simple to use in clinical practice regardless of differences in overall survival and local recurrence [5-7]. Therefore, an accurate preoperative evaluation is essential because individualized treatment measures are required for different local staging patients.

Diffusion-weighted imaging (DWI) could improve contrast between the lesion and normal tissue, which could improve the accuracy of rectal cancer detection. Compared with T2WI alone, T2WI combined with DWI has the best accuracy and specificity for the diagnosis of rectal cancer; moreover, that can improve accuracy of rectal cancer staging $[8,9]$.

Texture analysis (TA), as a new image postprocessing technology, can quantitatively describe tissue heterogeneity in medical images. About rectal cancer, there are many TA studies involved on T2WI and ADC maps. The study of Yang et al. [10] reported that T2WI histogram parameters could differentiate the positive and negative lymph node. TA derived from T2WI can be used for prediction of the 
TABLE 1: MRI sequences.

\begin{tabular}{|c|c|c|c|c|c|c|c|c|c|}
\hline MRI sequence & $\mathrm{TR}(\mathrm{ms})$ & $\mathrm{TE}(\mathrm{ms})$ & TSE factor & Slice thickness (mm) & Gap (mm) & FOV $(\mathrm{cm})$ & Matrix & NSA & $b$-value \\
\hline Sagittal T2WI & 3577 & 70 & 20 & 3 & 0 & $24 \times 24 \times 7.2$ & $300 \times 266$ & 3 & - \\
\hline Coronal T2WI & 3000 & 75 & 18 & 2 & 0 & $18 \times 18 \times 0.4$ & $300 \times 218$ & 3 & - \\
\hline Axial T2WI & 3000 & 75 & 18 & 3 & 0 & $18 \times 18 \times 7.2$ & $368 \times 273$ & 3 & - \\
\hline DWI & 2750 & 76 & - & 3 & 0 & $22 \times 22 \times 7.2$ & $112 \times 108$ & 2 & 0,1000 \\
\hline
\end{tabular}

TR: repetition time; TE: echo time; TSE: turbo spin echo; FOV: field of view; NSA: number of signal averaged.

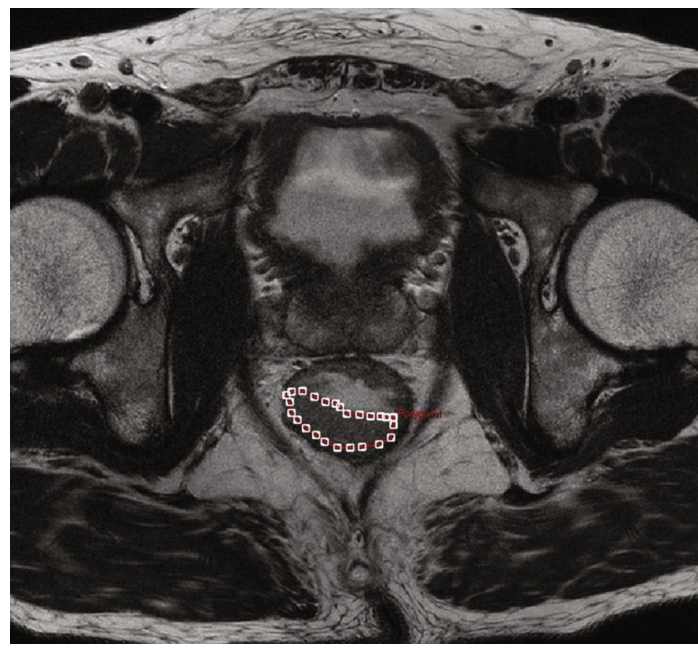

Figure 1: Example image for delineating a rectal lesion on T2WI.

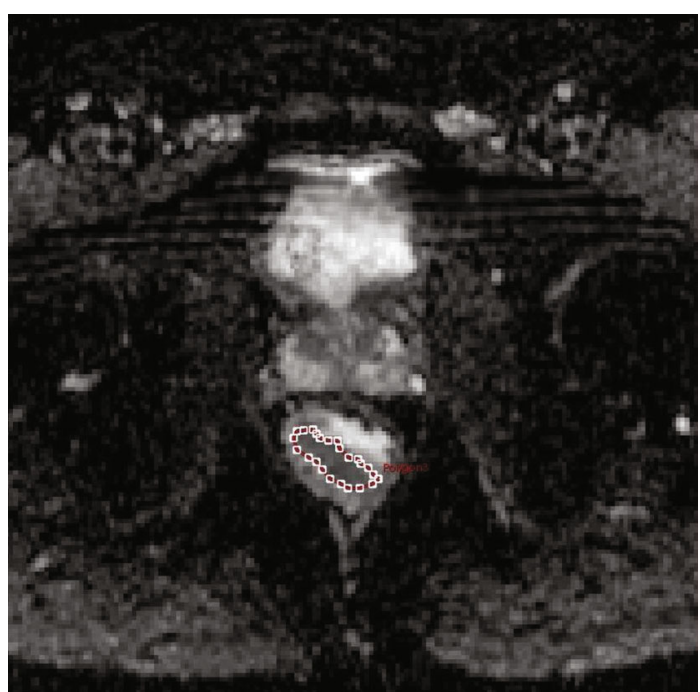

FIgURE 2: Example image for delineating a rectal lesion tumor edge on the ADC map.

rectal cancer $\mathrm{T}$ stage. [11]. In addition, our previous research results showed that TA from ADC maps could predict the local stage of rectal cancer [12].

In the present study, we investigated to compare TA from T2WI and ADC maps between different pathological $\mathrm{T}$ and $\mathrm{N}$ stages and to evaluate diagnostic performance to further confirm which TA analysis is better in diagnosis performance.
TABLe 2: Clinical and pathological data of patients.

\begin{tabular}{lc}
\hline Characteristics & No. of patients $(n=146)$ \\
\hline Gender & 86 \\
Male & 60 \\
Female & \\
Tumor location & 55 \\
Upper rectum & 66 \\
Middle rectum & 25 \\
Low rectum & \\
Differentiated grade & 28 \\
Well differentiated & 67 \\
Moderately differentiated & 51 \\
Poorly differentiated & \\
Pathological T stage & 8 \\
T1 & 37 \\
T2 & 25 \\
T3a & 54 \\
T3b & 17 \\
T3c & 5 \\
T4 & \\
Pathological N stage & \\
N0 & \\
N1 & \\
N2 & \\
\hline
\end{tabular}

\section{Materials and Methods}

2.1. Patients. This retrospective study was approved by the Institutional Review Board of Changshu Hospital of Soochow University. As this study has retrospective nature, requirements for written informed consent were waived. Inclusion criteria were (1) biopsy-proven nonmucinous adenocarcinoma and (2) the complete pathological $\mathrm{T}$ and $\mathrm{N}$ stage report. Exclusion criteria were (1) motion artifacts and magnetic sensitive artifacts affecting image quality and (2) treatment with preoperative chemotherapy or radiotherapy.

Finally, 146 patients were enrolled in the final study between October 2016 and October 2020.

2.2. $M R I$. All MRI examinations were performed on $3.0 \mathrm{~T}$ scanner (Intera Achieva TX, Philips Medical System) using a phased-array surface coil.

MR sequences included sagittal, oblique axial (perpendicular to the tumor axis), and oblique coronal (parallel to 
TABLe 3: Texture parameters between the pT1-3a and pT3b-4 stages.

\begin{tabular}{lccr}
\hline Texture parameters & pT1-3a $(n=70)$ & pT3b-4 $(n=76)$ & $p$ value \\
\hline T2WI & & & ICC \\
Skewness & $0.785 \pm 0.397$ & $0.871 \pm 0.384$ & 0.185 \\
Kurtosis & $1.109 \pm 0.425$ & $1.378 \pm 0.593$ & 0.002 \\
Energy $\left(\times 10^{-3}\right)$ & $0.685(0.580,0.871)$ & $0.515(0.452,0.605)$ & $<0.001$ \\
Entropy & $9.226(8.480,9.488)$ & $9.590(9.342,9.869)$ & $<0.001$ \\
Uniformity & $0.812 \pm 0.068$ & $0.798 \pm 0.073$ & 0.228 \\
Inertia & $159.572(126.822,195.283)$ & 0.913 \\
Correlation $\left(\times 10^{-3}\right)$ & $1.358 \pm 0.470$ & $163.749(125.463,203.394)$ & 0.905 \\
ADC & & $1.304 \pm 0.502$ & 0.865 \\
Skewness & $0.382 \pm 0.311$ & 0.838 \\
Kurtosis & $0.661 \pm 0.375$ & $0.516 \pm 0.360$ & 0.508 \\
Energy $\left(\times 10^{-3}\right)$ & $0.949(0.625,0.693)$ & $0.809 \pm 0.390$ & 0.018 \\
Entropy & $9.672 \pm 1.203$ & $0.690(0.583,1.445)$ & 0.020 \\
Uniformity & $0.761 \pm 0.057$ & $10.093 \pm 1.235$ & 0.875 \\
Inertia & $922.235(621.034,1189.230)$ & $0.769 \pm 0.070$ & 0.912 \\
Correlation $\left(\times 10^{-3}\right)$ & $0.448(0.349,0.638)$ & $737.177(520.237,1060.946)$ & 0.903 \\
\hline
\end{tabular}

TABle 4: Texture parameters between the pN0 and pN1-2 stages.

\begin{tabular}{lccc}
\hline Texture parameters & pN0 $(n=91)$ & $\mathrm{pN} 1-2(n=55)$ & $p$ value \\
\hline T2WI & $0.763 \pm 0.380$ & & ICC \\
Skewness & $1.253 \pm 0.552$ & $0.940 \pm 0.389$ & 0.007 \\
Kurtosis & $0.639(0.530,0.786)$ & $1.243 \pm 0.510$ & 0.911 \\
Energy $\left(\times 10^{-3}\right)$ & $9.342(8.788,9.662)$ & $0.516(0.437,0.613)$ & $<0.001$ \\
Entropy & $0.810 \pm 0.064$ & $9.537(9.135,9.773)$ & 0.856 \\
Uniformity & $163.749(125.463,203.394)$ & $0.797 \pm 0.081$ & 0.867 \\
Inertia & $1.341 \pm 0.473$ & $157.387(119.347,184.395)$ & 0.833 \\
Correlation $\left(\times 10^{-3}\right)$ & & $1.312 \pm 0.510$ & 0.915 \\
ADC & $0.403 \pm 0.283$ & 0.856 \\
Skewness & $0.682 \pm 0.346$ & $0.532 \pm 0.414$ & 0.729 \\
Kurtosis & $0.832(0.589,1.493)$ & $0.832 \pm 0.437$ & 0.863 \\
Energy $\left(\times 10^{-3}\right)$ & $9.863 \pm 1.212$ & $0.710(0.592,1.503)$ & 0.045 \\
Entropy & $0.762 \pm 0.067$ & $9.938 \pm 1.278$ & 0.033 \\
Uniformity & $830.845(529.492,1116.510)$ & $0.771 \pm 0.060$ & 0.747 \\
Inertia & $0.484(0.377,0.734)$ & 0.863 \\
Correlation $\left(\times 10^{-3}\right)$ & & 0.902 \\
\hline
\end{tabular}

the tumor axis) T2WI and oblique axial DWI (Table 1). The position and scanning parameters of oblique axial DWI were consistent with oblique axial T2WI. The ADC map is automatically generated by DWI with a $b$ value of 0 and $1000 \mathrm{~s} / \mathrm{mm}^{2}$.

2.3. Textural Feature Calculation. TA was performed on axial T2WI and ADC maps, respectively, using in-house software (Omni-Kinetics, GE Healthcare) by two authors (Zhihua $\mathrm{Lu}$ and Ming Li). The regions of interest (ROIs) involve as much tumor tissue as possible on the largest tumor slice, excluding necrosis, cysts, and gas (Figures 1 and 2). Then, texture features based on T2WI and ADC maps were calculated automatically. The seven texture features that we chose included skewness, kurtosis, and uniformity (first-order statistics) and entropy, energy, inertia, and correlation (secondorder statistics).

2.4. Histopathologic Analysis. A pathological T (pT) and N $(\mathrm{pN})$ staging report was performed based on the eighth edition of the American Joint Committee on Cancer (AJCC 8th) [13]. The histopathological report of local $\mathrm{T}$ and $\mathrm{N}$ staging was referred to as the gold standard in this study. 
TABLE 5: Performance of texture parameters to discriminate high-stage tumors.

\begin{tabular}{|c|c|c|c|c|c|c|}
\hline Parameters & AUC & $p$ value & $95 \% \mathrm{CI}$ & Sensitivity (\%) & Specificity (\%) & Cutoff value \\
\hline \multicolumn{7}{|c|}{ Discriminate $\mathrm{pT} 3 \mathrm{~b}-4$ stage } \\
\hline T2-kurtosis & 0.638 & 0.0028 & $0.555,0.716$ & 53.9 & 75.7 & $>1.332$ \\
\hline T2-energy & 0.793 & $<0.0001$ & $0.718,0.856$ & 60.5 & 87.1 & $\leq 0.528$ \\
\hline T2-entropy & 0.743 & $<0.0001$ & $0.664,0.811$ & 76.3 & 64.3 & $>9.329$ \\
\hline A-skewness & 0.605 & 0.0248 & $0.521,0.685$ & 30.3 & 91.4 & $>0.730$ \\
\hline A-kurtosis & 0.618 & 0.0121 & $0.534,0.697$ & 50.0 & 77.1 & $>0.818$ \\
\hline A-entropy & 0.602 & 0.0292 & $0.518,0.682$ & 75.0 & 42.9 & $>9.395$ \\
\hline \multicolumn{7}{|c|}{ Discriminate $\mathrm{pN} 1-2$ stage } \\
\hline T2-skewness & 0.640 & 0.0029 & $0.556,0.718$ & 70.9 & 54.9 & $>0.729$ \\
\hline T2-energy & 0.713 & $<0.0001$ & $0.632,0.784$ & 67.3 & 73.6 & $\leq 0.547$ \\
\hline T2-entropy & 0.602 & 0.0313 & $0.518,0.682$ & 72.7 & 48.4 & $>9.288$ \\
\hline A-skewness & 0.618 & 0.0243 & $0.535,0.698$ & 40.0 & 90.1 & $>0.729$ \\
\hline A-kurtosis & 0.601 & 0.0489 & $0.516,0.681$ & 29.1 & 93.4 & $>1.219$ \\
\hline
\end{tabular}

Notes: T2-: texture parameters from the T2WI map; A-: texture parameters from the ADC map; CI: confidence interval; AUC: area under the curve.

2.5. Statistical Analysis. First, the Kolmogorov-Smirnov test was used; data were presented as mean \pm standard deviation or median \pm interquartile range. To assess the difference of texture parameters between pT1-3a vs. pT3b-4 stage and pN0 vs. pN1-2 stage from the T2WI and ADC maps, the $t$-test or Mann-Whitney $U$ test was performed. Subsequent receiver operating characteristic (ROC) curve analysis was performed with those significant different parameters from the T2WI and ADC maps. The correlations between those significant different parameters from the T2WI and ADC maps and pathological $\mathrm{T}$ and $\mathrm{N}$ stages were used by the Spearman analysis. Intraclass correlation coefficients (ICCs) were calculated for interobserver agreements. SPSS and MedCalc software was used for statistical analysis. The significant level was set as $p$ value $\leq 0.05$.

\section{Results}

3.1. Clinical and Pathological Findings. 146 patients comprised 86 males (41-89 years; median, 68 years) and 60 females (41-84 years; median, 67 years). Detailed clinical and pathological data are listed in Table 2. According to the design of this study, 70 patients were grouped as pT1$3 \mathrm{a}$ and 76 patients were grouped as $\mathrm{pT} 3 \mathrm{~b}-4.91$ patients were grouped as $\mathrm{pN} 0$, and 55 patients were grouped as pN1-2.

3.2. Texture Parameters in Differentiating pT1-3a and $p T 3 b$ 4 Stages. On T2WI, the pT3b-4 stage tumor demonstrated significantly higher kurtosis and entropy and lower energy than the pT1-3a stage tumor (all $p<0.05$, Table 3 ). On the ADC map, the pT3b-4 stage tumor demonstrated significantly higher skewness, kurtosis, and entropy than the pT1-3a stage tumor (all $p<0.05$ ). No significant differences were found in other TA parameters between different $\mathrm{T}$ stages. The results of ICCs are shown in Table 3.

3.3. Texture Parameters in Differentiating pNO and pN1-2 Stages. On T2WI, the pN1-2 stage tumor demonstrated significantly higher skewness and entropy and lower energy

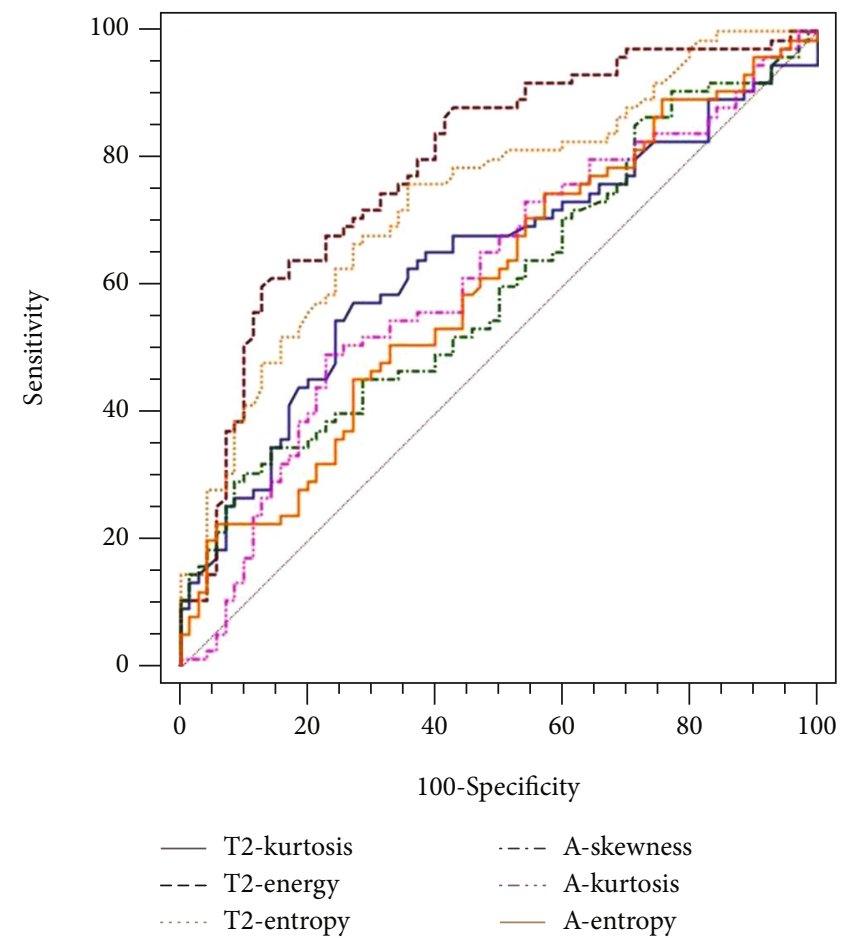

FIgURE 3: The ROC map of texture parameters in differentiating pT3b-4 from the pT1-3a stage.

than the pN0 stage tumor (all $p<0.05$, Table 4). On the ADC map, the pN1-2 stage tumor demonstrated significantly higher skewness and kurtosis than the pN0 stage tumor (all $p<0.05$ ). No significant differences were found in other TA parameters between different $\mathrm{N}$ stages. The results of ICCs are shown in Table 4.

3.4. Performance of Texture Parameters to Distinguish the pT3b-4 and pN1-2 Stages. For differentiating pT3b-4 from pT1-3a stage tumors, energy derived from T2WI had the largest AUC (79.3\%). Kurtosis derived from the ADC map 
TABLE 6: Correlations between significant different parameters from the T2WI and ADC maps and pathological T and N stages.

\begin{tabular}{lccccc}
\hline \multirow{2}{*}{ Parameters } & \multicolumn{2}{c}{ Pathological T stage } & \multirow{2}{*}{ Parameters } & \multicolumn{2}{c}{ Pathological N stage } \\
& $R_{\mathrm{s}}$ & $p$ value & T2-skewness & 0.229 & 0.005 \\
T2-kurtosis & 0.266 & 0.001 & T2-energy & -0.359 & $<0.001$ \\
T2-energy & -0.494 & $<0.001$ & T2-entropy & 0.175 & 0.035 \\
T2-entropy & 0.445 & $<0.001$ & A-skewness & 0.196 & 0.018 \\
A-skewness & 0.199 & 0.016 & A-kurtosis & 0.168 & 0.043 \\
A-kurtosis & 0.235 & 0.004 & & \\
A-entropy & 0.221 & 0.007 & & \\
\hline
\end{tabular}

Notes: T2-: texture parameters from the T2WI map; A-: texture parameters from the ADC map.

had the largest AUC (61.8\%) (Table 5, Figure 3). Furthermore, energy derived from T2WI showed a better correlation with the pathological $\mathrm{T}$ stage $\left(R_{\mathrm{s}}=-0.494\right)$ than kurtosis derived from ADC maps (Table 6).

For differentiating pN1-2 from pN0 stage tumors, energy derived from T2WI had the largest AUC (71.3\%). Skewness derived from ADC maps had the largest AUC (61.8\%) (Figure 4). Furthermore, energy derived from T2WI showed a better correlation with the pathological $\mathrm{N}$ stage $\left(R_{\mathrm{s}}=-0.359\right)$ than skewness derived from ADC maps (Table 6).

\section{Discussion}

The present study showed that kurtosis, energy, and entropy from T2WI and skewness, kurtosis, and entropy from ADC maps could significantly differentiate pT1-3a and pT3b-4 stages. In addition, we found that skewness, energy, and entropy from T2WI and skewness and kurtosis from ADC maps could significantly differentiate $\mathrm{pN} 0$ and $\mathrm{pN} 1-2$ stages. Among the aforementioned significant different parameters, those parameters derived from T2WI had higher AUC differentiating pT3b-4 and pN1-2 stage tumors than those derived from ADC maps. Furthermore, those parameters derived from T2WI showed a better correlation with pathological $\mathrm{T}$ and $\mathrm{N}$ stages than those derived from ADC maps. The results indicate that the diagnostic performance of TA parameters derived from T2WI was better than that derived from ADC maps.

High-resolution T2WI plays a key point in the local stage of rectal cancer. In recent years, some studies applied TA derived from T2WI in order to improve the preoperative stage and predict prognostic factors of rectal cancer $[10,11$, 14]. In our study, the pT3b-4 stage tumor demonstrated higher kurtosis and entropy and lower energy than the pT1-3a stage tumor. In addition, the pN1-2 stage tumor demonstrated higher skewness and entropy and lower energy than the pN0 stage tumor. Theoretically, higher entropy and lower energy reflect higher heterogeneity of the lesion [15]. This may interpret our results. Yang et al. [10] have reported that the lymph node-positive group had a significant lower energy and higher entropy than the lymph node-negative group.

DWI could improve contrast between the lesion and normal tissue. It has been widely used in preoperative imag-

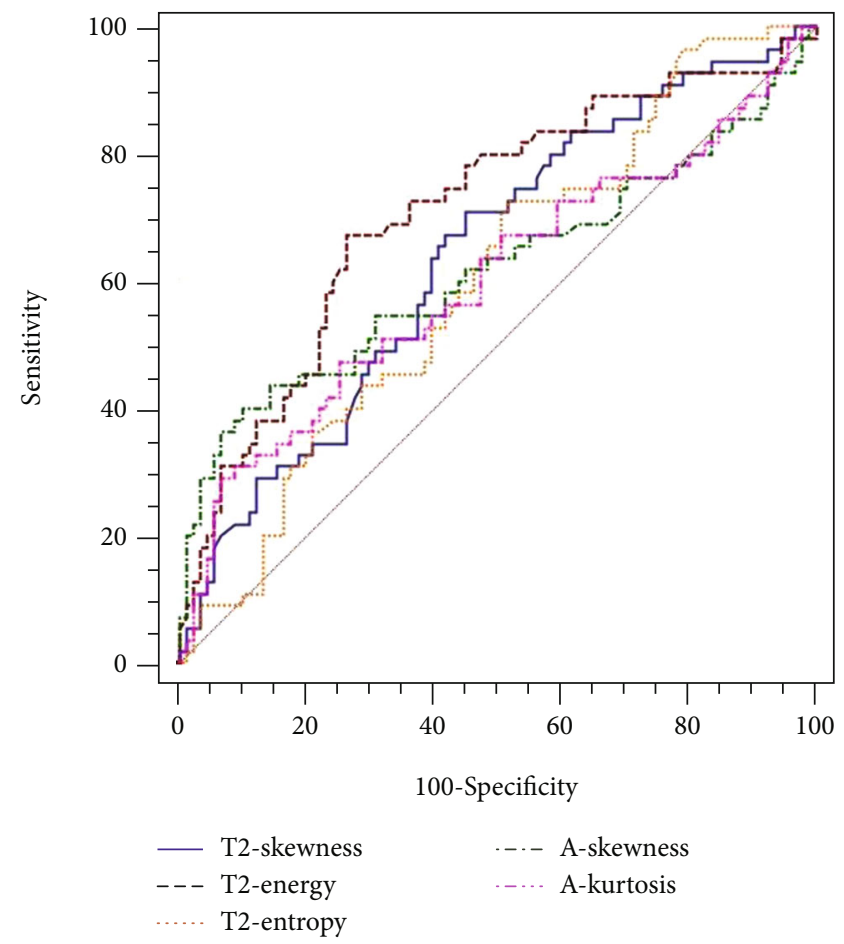

FIgURE 4: The ROC map of texture parameters in differentiating pN1-2 from the pN0 stage.

ing of rectal cancer because T2WI combined with DWI can improve the accuracy of rectal cancer staging [8]. Our previous research and other studies have shown that the ADC value estimated from DWI was related to tumor aggressiveness [16-18]. TA parameters derived from ADC maps were more sensitive and reliable markers for the accurate operative stage and evaluate the efficacy to treatment in rectal cancer $[12,19]$. In our study, the pT3b-4 stage tumor demonstrated higher skewness, kurtosis, and entropy than the pT1-3a stage tumor. In addition, the pN1-2 stage tumor demonstrated higher skewness and kurtosis than the pN0 stage tumor. Higher value of skewness and kurtosis reflects more complexity and heterogeneity in tumors. Our result concluded that skewness and kurtosis derived from ADC maps as imaging biomarkers might be differentiating the positive lymph node of rectal cancer.

ROC curves showed that significant different parameters derived from T2WI had higher AUC differentiating pT3b-4 
and pN1-2 stage tumors than those derived from ADC maps. Energy was shown to be the best performance in predicting pT3b-4 and pN1-2 stage tumors among TA parameters with yielding AUC of $79.3 \%$ and $71.3 \%$, respectively. Furthermore, the Spearman correlation analysis showed that energy derived from T2WI had the better correlation with pathological $\mathrm{T}$ and $\mathrm{N}$ stages than parameters derived from the ADC map. Therefore, our results indicated that the diagnostic performance of TA parameters derived from T2WI was better than that derived from ADC maps. The reason may be that high-resolution T2WI can reflect much more details in the image of the lesion. In addition, T2WI is the preferred sequence for the preoperative stage of rectal cancer. Thus, TA derived from T2WI may be a more useful tool to aid radiologists and surgeons in selecting treatment.

There are some limitations. First, rectal cancer often grows infiltrating along the bowel wall. Lesions and normal bowel walls are often unclear; then, the measurement cannot accurately delineate the entire tumor. So, the measurement in our study was performed on a single slice of the largest tumor slice. Second, we evaluated seven TA parameters in our study because these parameters have been proven by previous studies to be related to the heterogeneity of the disease. No other more parameters were included for the group study. Third, we only included pathological $\mathrm{T}$ and $\mathrm{N}$ stage as the grouping standard. More prognostic factors should be included in the later period for further research.

In conclusion, our study showed that the diagnostic performance of TA parameters derived from T2WI was better than that derived from ADC maps. In particular, the energy derived from T2WI was the optimal parameter for diagnostic efficiency. Thus, TA derived from T2WI may be a more useful tool to aid radiologists and surgeons in selecting treatment.

\section{Data Availability}

The data used to support the findings of this study are available from the corresponding author upon request.

\section{Conflicts of Interest}

The authors declare no conflicts of interest.

\section{Authors' Contributions}

Ming Li and Xiaodan Xu contributed equally to this work.

\section{Acknowledgments}

This work was supported in part by the Jiangsu Provincial Medical Youth Talent under Grant QNRC2016212, Jiangsu Committee of Health under Grant H2018071, Suzhou Clinical Special Disease Diagnosis and Treatment Program under Grant LCZX20182, Suzhou GuSu Medical Talent Project under Grants GSWS2019077 and GSWS2020108, and Suzhou Science and Technology Development Program under Grant SYS2020058.

\section{References}

[1] National Health Commission of the People's Republic of China, "Chinese protocol of diagnosis and treatment of colorectal cancer (2020 edition)," Chinese Journal of Digestive Surgery, vol. 19, no. 6, pp. 563-588, 2020.

[2] H. J. Schmoll, E. van Cutsem, A. Stein et al., "ESMO consensus guidelines for management of patients with colon and rectal cancer. A personalized approach to clinical decision making," Annals of Oncology, vol. 23, no. 10, pp. 2479-2516, 2012.

[3] Oncology Branch of the Chinese Medical Association, Medical and Hospital Administration Bureau of the Health and Family Planning Commission of the People's Republic of China, "Chinese standards for diagnosis and treatment of colorectal cancer (2017 edition) (in Chinese)," Chinese Journal of Gastrointestinal Surgery, vol. 21, pp. 92-106, 2018.

[4] R. Glynne-Jones, L. Wyrwicz, E. Tiret et al., "Corrections to "Rectal cancer: ESMO clinical practice guidelines for diagnosis, treatment and follow-up"," Annals of Oncology, vol. 29, article iv263, Supplement 4, 2018.

[5] R. Zinicola, G. Pedrazzi, N. Haboubi, and R. J. Nicholls, "The degree of extramural spread of T3 rectal cancer: an appeal to the American Joint Committee on Cancer," Colorectal Disease, vol. 19, no. 1, pp. 8-15, 2017.

[6] S. Merkel, K. Weber, V. Schellerer et al., "Prognostic subdivision of ypT3 rectal tumours according to extension beyond the muscularis propria," The British Journal of Surgery, vol. 101, no. 5, pp. 566-572, 2014.

[7] H. Kaur, H. Choi, Y. N. You et al., "MR imaging for preoperative evaluation of primary rectal cancer: practical considerations," Radiographics, vol. 32, no. 2, pp. 389-409, 2012.

[8] G. N. Cong, M. W. Qin, H. You et al., "Diffusion weighted imaging combined with magnetic resonance conventional sequences for the diagnosis of rectal cancer," Zhongguo Yi Xue Ke Xue Yuan Xue Bao. Acta Academiae Medicinae Sinicae, vol. 31, no. 2, pp. 200-205, 2009.

[9] A. Delli Pizzi, D. Caposiena, D. Mastrodicasa et al., “Tumor detectability and conspicuity comparison of standard b1000 and ultrahigh b2000 diffusion-weighted imaging in rectal cancer," Abdominal Radiology, vol. 44, no. 11, pp. 3595-3605, 2019.

[10] L. Yang, D. Liu, X. Fang et al., "Rectal cancer: can T2WI histogram of the primary tumor help predict the existence of lymph node metastasis?," European Radiology, vol. 29, no. 12, pp. 6469-6476, 2019.

[11] H. C. Lu, F. Wang, and J. D. Yin, "Texture analysis based on sagittal fat-suppression and transverse T2-weighted magnetic resonance imaging for determining local invasion of rectal cancer," Frontiers in Oncology, vol. 10, p. 1476, 2020.

[12] Z. Lu, L. Wang, K. Xia et al., "Prediction of clinical pathologic prognostic factors for rectal adenocarcinoma: volumetric texture analysis based on apparent diffusion coefficient maps," Journal of Medical Systems, vol. 43, no. 12, p. 331, 2019.

[13] M. B. Amin, S. Edge, F. Greene et al., AJCC Cancer Staging Manual, Springer, New York, 8th edition, 2016.

[14] A. Delli Pizzi, A. M. Chiarelli, P. Chiacchiaretta et al., "MRIbased clinical-radiomics model predicts tumor response before treatment in locally advanced rectal cancer," Scientific Reports, vol. 11, no. 1, p. 5379, 2021.

[15] R. Duvauferrier, J. Bezy, V. Bertaud, G. Toussaint, J. Morelli, and J. Lasbleiz, "Texture analysis software: integration with a 
radiological workstation," Studies in Health Technology and Informatics, vol. 180, pp. 1030-1034, 2012.

[16] L. Curvo-Semedo, D. M. Lambregts, M. Maas, G. L. Beets, F. Caseiro-Alves, and R. G. Beets-Tan, "Diffusion-weighted MRI in rectal cancer: apparent diffusion coefficient as a potential noninvasive marker of tumor aggressiveness," Journal of Magnetic Resonance Imaging, vol. 35, no. 6, pp. 1365-1371, 2012.

[17] E. M. Hecht, M. Z. Liu, M. R. Prince et al., "Can diffusionweighted imaging serve as a biomarker of fibrosis in pancreatic adenocarcinoma?," Journal of Magnetic Resonance Imaging, vol. 46, no. 2, pp. 393-402, 2017.

[18] E. Nerad, A. Delli Pizzi, D. M. J. Lambregts et al., "The apparent diffusion coefficient (ADC) is a useful biomarker in predicting metastatic colon cancer using the ADC-value of the primary tumor," PLoS One, vol. 14, no. 2, article e0211830, 2019.

[19] L. Yang, M. Qiu, C. Xia et al., "Value of high-resolution DWI in combination with texture analysis for the evaluation of tumor response after preoperative chemoradiotherapy for locally advanced rectal cancer," AJR. American Journal of Roentgenology, vol. 12, pp. 1-8, 2019. 